\title{
IMMIGRAZIONE E NUOVE DISEGUAGLIANZE
}

\author{
Introduzione al tema monografico \\ di Bruno Anastasia e Maurizio Gambuzza
}

La crisi nord africana rappresenta solo l'ultimo di una serie di eventi, frequenti e purtroppo spesso drammatici, che ripropongono all'attenzione dell'opinione pubblica il tema dell'emigrazione dai paesi meno sviluppati e da quelli sottoposti a regimi illiberali. Anche per effetto del loro forte impatto mediatico, oltre che per l'utilizzo che di essi viene fatto nel confronto politico nel nostro Paese, la connotazione emergenziale che contraddistingue questi accadimenti tende ad essere traslata e a permeare il dibattito sulla questione più generale nella quale essi si inscrivono: l'immigrazione ed il suo possibile (e problematico) governo. Il tema della presenza straniera in Italia costituisce un argomento rovente, dunque, che coinvolge e preoccupa; che in alcuni suscita sentimenti di empatia con le popolazioni interessate e invita a mettere in campo iniziative volte all'ospitalità, alla solidarietà e all'accoglienza, mentre in altri - all'opposto - alimenta orientamenti di diffidenza, di timore e finanche di paura che inducono alla chiusura o all'intolleranza e finiscono per alimentare quell' "indurimento del carattere" che secondo Marco Revelli (Poveri, noi, Einaudi, 2010) costituisce uno dei tratti socio-culturali della società italiana contemporanea. Ingredienti che certo non contribuiscono a rendere la questione migrazioni più facile da delimitare, da comprendere e da trattare. Quanto mai utile, allora, aprire spazi per il confronto e la discussione anche sulla nostra rivista, come proposto in questo numero.

Il contrasto agli sbarchi e gli accordi con i paesi della sponda mediterranea rappresentano momenti utili per la lotta al traffico illegale che sfrutta il dramma della miseria e della disperazione di molti migranti, ma non aiutano ad affermare una risposta ed un orientamento corretto ai problemi che abbiamo di fronte: come ha sottolineato di recente Ambrosini (in www.lavoce.info del 15.2.2011) "l'etichetta di clandestini, applicata senza distinzione ai migranti che arrivano dal mare senza le prescritte autorizzazioni, appare una sorta di criminalizzazione preventiva di massa" perché nasconde frequentemente richiedenti asilo legittimi e cela l'esigenza di definire strategie umanitarie di accoglienza dei rifugiati (giusto per un problema di corretta collocazione 
dell'Italia nel panorama europeo, da questo punto di vista, è bene ricordare che il nostro Paese ospita 55mila rifugiati contro, ad esempio, i 593mila della Germania).

Che dietro problemi definitori e terminologici si nascondano spesso nodi irrisolti lo si ritrova anche in riferimento alla massa ben più consistente degli ingressi nel nostro Paese. L' "irregolarità" più che l'eccezione rappresenta la regola che governa i movimenti di immigrazione e, pertanto, una lampante testimonianza della difficoltà di gestire l'afflusso di immigrati senza che si alimentino i circuiti illegali di presenza e di lavoro. Un problema comune a tutti i Paesi occidentali e tutt'altro che di semplice soluzione: ma certamente dovrebbe far riflettere il fatto che proprio i paesi con una legislazione più restrittiva sulla carta come Spagna e Italia - siano stati nell'ultimo decennio i destinatari dei flussi quantitativamente più rilevanti.

La strada fin qui perseguita è insoddisfacente sia nella versione precedente la grande regolarizzazione del 2002 (quando le quote annue di ingressi consentiti erano irrisorie e prevalentemente per lavoro stagionale, così da indurre la formazione di un'ampia area di presenza e lavoro irregolari da sanare ex post) sia nella versione successiva, quando l'allargamento delle quote, come accaduto nel 2007-08, si è confrontato con un numero straordinariamente elevato di domande, tanto da non riuscire a gestirle in tempi ragionevoli; sia, infine, nella recente esperienza del decreto flussi gestito attraverso il meccanismo del cosiddetto click-day, quando a fronte dei circa 100mila "ingressi" in palio sono state presentate oltre 400mila domande (per lo più per lavoro domestico e colf, da parte di datori di lavoro stranieri). Generando, quindi, macroscopiche distorsioni e complicazioni gestionali, tanto per i migranti che per datori di lavoro e famiglie, e finendo così per tollerare la persistenza di aree di lavoro nero come conseguenza inevitabile di procedure di gestione degli ingressi estremamente lunghe, incerte e sottoposte a condizioni di estrema casualità degli esiti.

Se i decreti flussi finiscono soprattutto per regolarizzare persone già presenti sul territorio nazionale più che gestire gli ingressi veri e propri, l'evoluzione del fenomeno migratorio nel nostro Paese testimonia un avanzamento del processo di stabilizzazione e tendenziale integrazione, secondo una direzione opposta a quella auspicata dalla "Bossi-Fini" e dal "pacchetto-sicurezza", tendente a incoraggiare la cosiddetta immigrazione circolare.

Gli stranieri non rappresentano una componente marginale né, tanto meno, transitoria. Il profilo dei 4.235.059 stranieri residenti in Italia al gennaio 2010 (il 7\% della popolazione complessiva) mostra che oltre la metà di essi è titolare di uno status giuridico forte: o perché cittadini comunitari (notevolmente aumentati negli ultimi anni a seguito dell'allargamento dell'Unione europea) o perché titolari del permesso di soggiorno di lungo periodo (documento che si ottiene normalmente dopo cinque anni di residenza in Italia e apre la strada all'eventuale richiesta di cittadinanza italiana dopo ulteriori cinque anni). 
La loro dinamica di crescita è risultata negli ultimi anni particolarmente sostenuta; sempre considerando i residenti, nel corso del 2009 essi sono aumentati di 343mila unità $(+8,8 \%)$ mentre nei due anni precedenti l'incremento era stato ancora più consistente e aveva sfiorato le 500mila unità/annue per effetto degli ingressi dalla Romania.

Le previsioni demografiche disponibili, inoltre, indicano un trend chiaro di crescita; le più recenti rilasciate dall' Istat propongono tre ipotesi di scenario: in quella "bassa" si adotta un saldo migratorio netto attorno alle 150.000 persone all'anno; in quella "centrale" esso sale a 200.000; in quella "alta" si arriva a 250.000. Ciononostante in tutti e tre questi scenari il saldo migratorio non è sufficiente a garantire la stabilità della quota di popolazione 15-64 anni sul totale, né lo stesso attuale ammontare assoluto della popolazione in età lavorativa. E dunque anche questi flussi (consistenti) di immigrazione netta dovranno essere combinati con una crescita della partecipazione perché sia sostenibile l'assetto sociale ed economico italiano.

Questi scenari, difficilmente eludibili, offrono indicazioni su quello che sarà l'effettivo fabbisogno del mercato del lavoro italiano, mentre la realtà di una presenza ormai radicata nel nostro territorio invita ad una più attenta lettura dei meccanismi con cui questa si dispiega, delle diseguaglianze e delle forme di segregazione e marginalizzazione (occupazionale, spaziale, sociale) cui dà luogo, della qualità dell'integrazione che siamo in grado di mettere in campo.

Pur con tutte le carenze imputabili ad una strategia migratoria improntata ad una logica "di difesa", dove anche le sanatorie rispondono ad una funzione strutturale, l'Italia ha retto alla significativa crescita dell' afflusso dei cittadini stranieri senza che si generassero diffusi fenomeni di marginalizzazione e conflittualità. Il recente peggioramento generalizzato della situazione economica indotto dalla lunga crisi che anche il nostro Paese sta attraversando corre il rischio di interrompere questi esiti positivi in fin dei conti inaspettati? La carenza, non si sa fino a quando temporanea, di posti di lavoro innescherà fenomeni di competizione con l'offerta nazionale fin ad ora quasi sconosciuti?

I tumultuosi cambiamenti in atto nel continente africano sono un segnale che obbliga a guardare ai fenomeni migratori non solo come una risposta ai bisogni di forza lavoro delle società ricche ma soprattutto come una domanda di progressivo allargamento dei processi di sviluppo economico e di emancipazione sociale e civile.

I problemi legati alla presenza straniera risultano quindi particolarmente complessi e intorno ad essi si sono prodotte analisi diverse e in molti casi contrapposte in merito alle soluzioni possibili e praticabili. In questo numero presentiamo i contributi di Fabio Perocco, Rossana Cillo e Francesco Della Puppa, esponenti del Laboratorio di ricerca sull'immigrazione e sulle trasformazioni sociali dell'Università Ca' Foscari di Venezia, augurandoci che il punto di vista proposto apra a una discussione sul tema. 